\title{
CheckMate 025 Randomized Phase 3 Study: Outcomes by Key Baseline Factors and Prior Therapy for Nivolumab Versus Everolimus in Advanced Renal Cell Carcinoma
}

\author{
Bernard Escudier $^{a, *}$, Padmanee Sharma $^{b}$, David F. McDermott $^{c}$, Saby George ${ }^{d}$, \\ Hans J. Hammers ${ }^{e, 1}$, Sandhya Srinivas ${ }^{f}$, Scott S. Tykodi ${ }^{g}$, Jeffrey A. Sosman ${ }^{h, 2}$, \\ Giuseppe Procopio ${ }^{i}$, Elizabeth R. Plimack ${ }^{j}$, Daniel Castellano $^{k}$, Howard Gurney $^{l}$, \\ Frede Donskov $^{m}$, Katriina Peltola ${ }^{n}$, John Wagstaff $^{o}$, Thomas C. Gauler ${ }^{p}$, Takeshi Ueda ${ }^{q}$, \\ Huanyu Zhao $^{r}$, Ian M. Waxman ${ }^{r}$, Robert J. Motzer ${ }^{s}$, \\ on behalf of the CheckMate 025 investigators
}

\begin{abstract}
${ }^{a}$ Gustave Roussy, Villejuif, France; ${ }^{\mathrm{b}}$ MD Anderson Cancer Center, University of Texas, Houston, TX, USA; ${ }^{\mathrm{c}}$ Beth Israel Deaconess Medical Center, Dana-Farber/ Harvard Cancer Center, Boston, MA, USA; ${ }^{\mathrm{d}}$ Roswell Park Cancer Institute, Buffalo, NY, USA; ${ }^{\mathrm{e} J o h n s ~ H o p k i n s ~ S i d n e y ~ K i m m e l ~ C o m p r e h e n s i v e ~ C a n c e r ~ C e n t e r, ~}$ Baltimore, MD, USA; ${ }^{\mathrm{f}}$ Stanford Cancer Institute, Stanford, CA, USA; ${ }^{\mathrm{g}}$ University of Washington and Fred Hutchinson Cancer Research Center, Seattle, WA, USA; ${ }^{\mathrm{h}}$ Vanderbilt University Medical Center, Nashville, TN, USA; ${ }^{\mathrm{i}}$ Fondazione Istituto Nazionale Tumori, Milan, Italy; ${ }^{\mathrm{j}}$ Fox Chase Cancer Center, Philadelphia, PA, USA; ${ }^{k}$ Hospital Universitario 12 De Octubre, Madrid, Spain; ${ }^{1}$ Westmead Hospital, Westmead, NSW, Australia; ${ }^{\mathrm{m}}$ Aarhus University Hospital, Aarhus, Denmark; ${ }^{\mathrm{n}}$ Comprehensive Cancer Center, Helsinki University Central Hospital Cancer Center, Helsinki, Finland; ${ }^{\circ}$ South West Wales Cancer Institute and Swansea University College of Medicine, Swansea, UK; ${ }^{\mathrm{p}}$ University Hospital Essen of University of Duisburg-Essen, Essen, Germany; ${ }^{\mathrm{q}}$ Chiba Cancer Center, Chiba, Japan; ${ }^{\mathrm{r}}$ Bristol-Myers Squibb, Princeton, NJ, USA; ${ }^{\mathrm{S}}$ Memorial Sloan Kettering Cancer Center, New York, NY, USA
\end{abstract}

\section{Article info}

\section{Article history:}

Accepted February 7, 2017

Associate Editor:

Giacomo Novara

Keywords:

Everolimus

Immune checkpoint inhibitor

Nivolumab

Phase 3

Renal cell carcinoma

\section{EU ACME}

www.eu-acme.org/

europeanurology

Please visit www.eu-acme.org/

europeanurology to read and

answer questions on-line.

\begin{abstract}
Background: The randomized, phase 3 CheckMate 025 study of nivolumab $(n=410)$ versus everolimus $(n=411)$ in previously treated adults (75\% male; $88 \%$ white) with advanced renal cell carcinoma (aRCC) demonstrated significantly improved overall survival (OS) and objective response rate (ORR).

Objective: To investigate which baseline factors were associated with OS and ORR benefit with nivolumab versus everolimus.

Design, setting, and participants: Subgroup OS analyses were performed using KaplanMeier methodology. Hazard ratios were estimated using the Cox proportional hazards model.

Intervention: Nivolumab $3 \mathrm{mg} / \mathrm{kg}$ every $2 \mathrm{wk}$ or everolimus $10 \mathrm{mg}$ once daily.

Results and limitations: The minimum follow-up was 14 mo. Baseline subgroup distributions were balanced between nivolumab and everolimus arms. Nivolumab demonstrated an OS improvement versus everolimus across subgroups, including Memorial Sloan Kettering Cancer Center (MSKCC) and International Metastatic Renal Cell Carcinoma Database Consortium risk groups; age $<65$ and $\geq 65 \mathrm{yr}$; one and two or more sites of metastases; bone, liver, and lung metastases; number of prior therapies; duration of

1 Current affiliation: UT Southwestern-Kidney Cancer Program, Dallas, TX, USA.

2 Current affiliation: Robert Lurie Comprehensive Cancer Center of Northwestern University, Chicago, IL, USA.

* Corresponding author. Gustave Roussy, 114 Rue Edouard Vaillant, 94800 Villejuif, France. Tel. +33 142115410 .

E-mail address: escudier@gustaveroussy.fr (B. Escudier).
\end{abstract}


prior therapy; and prior sunitinib, pazopanib, or interleukin-2 therapy. The benefit with nivolumab versus everolimus was noteworthy for patients with poor MSKCC risk (hazard ratio $0.48,95 \%$ confidence interval $0.32-0.70$ ). The mortality rate at 12 mo for all subgroups was lower with nivolumab compared with everolimus. ORR also favored nivolumab. The incidence of grade 3 or 4 treatment-related adverse events across subgroups was lower with nivolumab. Limitations include the post hoc analysis and differing sample sizes between groups.

Conclusion: The trend for OS and ORR benefit with nivolumab for multiple subgroups, without notable safety concerns, may help to guide treatment decisions, and further supports nivolumab as the standard of care in previously treated patients with aRCC.

Patient summary: We investigated the impact of demographic and pretreatment features on survival benefit and tumor response with nivolumab versus everolimus in advanced renal cell carcinoma (aRCC). Survival benefit and response were observed for multiple subgroups, supporting the use of nivolumab as a new standard of care across a broad range of patients with previously treated aRCC.

The trial is registered on ClinicalTrials.gov as NCT01668784.

(C) 2017 European Association of Urology. Published by Elsevier B.V. All rights reserved.

\section{Introduction}

As the treatment paradigm for advanced renal cell carcinoma (aRCC) shifts in response to the development and approval of new therapies, a deeper understanding of patient baseline factors and/or disease characteristics affecting clinical outcomes is necessary and may help in guiding treatment decisions. Prognostic models for RCC have been developed that incorporate factors such as performance status, time from diagnosis to treatment, hemoglobin, calcium and lactate dehydrogenase concentrations, and neutrophil and platelet counts $[1,2]$. These models are limited because they were developed before the advent of modern immunotherapies and they do not include other factors that have also been shown to be associated with prognosis, such as the number and duration of prior therapies, sites of metastases, and age [3-6]. Further investigation of prognostic factors is needed for the development of risk models that more accurately reflect the current treatment landscape.

The phase 3 CheckMate 025 study in previously treated patients with aRCC demonstrated superior overall survival (OS) with nivolumab compared with everolimus [7]. Median OS was 25.0 mo (95\% confidence interval [CI] 21.8-not reached [NR]) for nivolumab versus $19.6 \mathrm{mo}$ (95\% CI 17.6-23.1) for everolimus. The investigatorassessed objective response rate (ORR) was $25 \%$ versus $5 \%(p<0.001)[7]$, while the confirmed ORR was $22 \%$ versus $4 \%$ [8]. Treatment with nivolumab also provided an OS benefit versus everolimus across prespecified subgroups of patients, including those with different Memorial Sloan Kettering Cancer Center (MSKCC) risk, number of prior antiangiogenic therapies, geographical region, age, and sex. [7].

The objectives of this analysis were to investigate further whether the OS and ORR benefits observed with nivolumab versus everolimus in the overall population were also observed in patients with poor prognostic baseline disease, and if demographic and pretreatment characteristics, including prior therapy, with an impact on outcomes with nivolumab can be identified.

\section{Patients and methods}

\subsection{Patients}

Adults with histological confirmation of aRCC with a clear-cell component were eligible. Additional eligibility criteria were reported previously [7]. Subgroups of patients were analyzed according to the following characteristics at baseline: MSKCC risk score (favorable, intermediate, poor) [2], International Metastatic Renal Cell Carcinoma Database Consortium (IMDC) risk score (favorable, intermediate, poor), age ( $<65$ and $\geq 65 \mathrm{yr}$ ), number ( 1 and $>1$ ) and sites (bone, liver, lung) of metastases, prior therapy (sunitinib, pazopanib, interleukin-2), duration of first-line therapy ( $<6$ and $\geq 6 \mathrm{mo}$ ), and number of prior therapies ( 1 or 2 ). Analyses are based on data collected via a case report form (data collected from an interactive voice response system was used in the previous publication) [7].

\subsection{Study design and treatments}

This was a phase 3, randomized, open-label study of nivolumab versus everolimus. The detailed study design was described previously [7]. Patients were randomized $1: 1$ to receive nivolumab $3 \mathrm{mg} / \mathrm{kg}$ intravenously for $60 \mathrm{~min}$ every $2 \mathrm{wk}$ or an everolimus $10-\mathrm{mg}$ tablet orally once daily.

\subsection{Endpoints and assessments}

The primary endpoint was OS, defined as time from randomization to death; the key secondary endpoint was investigator-assessed ORR, defined as the number of patients with complete response or partial response divided by the number of randomized patients. Disease assessments (per Response Evaluation Criteria in Solid Tumors [RECIST] v1.1) [9] were performed using computed tomography or magnetic resonance imaging at baseline and every $8 \mathrm{wk}$ following randomization for the first year, then every 12 wk until progression or treatment discontinuation. Safety was assessed at each clinic visit. Subgroups reported here were assessed for OS, ORR, and safety.

\subsection{Study oversight}

This study was approved by the institutional review board or independent ethics committee at each center and conducted in accordance with Good Clinical Practice guidelines defined by the International Conference on 
Harmonisation. All patients provided written informed consent to participate according to the principles of the Declaration of Helsinki.

\subsection{Statistical analyses}

Descriptive statistics for categorical variables are reported as frequencies and percentages. OS was estimated using the Kaplan-Meier productlimit methods [10]; median OS and 95\% CI were reported using Brookmeyer and Crowley methods [11]; 95\% CIs were constructed via $\log$-log transformation. Hazard ratios for nivolumab versus everolimus were estimated using the Cox proportional hazards model with treatment group as a single covariate [12]. Unstratified hazard ratios and corresponding 95\% CIs were used to generate a forest plot of OS comprising each subgroup. A forest plot of the unweighted differences in ORR between nivolumab and everolimus and corresponding $95 \% \mathrm{Cl}$ using the Newcombe approach was produced across subgroups [13]. To assess whether the relationship between OS and treatment differed by various patient characteristics of interest, we separately tested the interaction between treatment and each baseline characteristic using a Cox proportional hazards model. Similarly, for ORR and treatment-related adverse event (TRAE) rates, the interaction between treatment and each baseline characteristic was tested using a logistic regression model. Continuous variables were only used for the interaction test for age (in years) and duration of prior therapy (in months); for all other subgroups, categorical variables were used. The analyses were conducted using SAS version 9 software (SAS Institute Inc., Cary, NC, USA).

\section{Results}

\subsection{Patients}

The analyses included 410 and 411 patients randomized to nivolumab and everolimus, respectively, between October 2012 and March 2014 (Supplementary Fig. 1). The distribution of patients in each subgroup was balanced between nivolumab and everolimus (Table 1 ). The minimum follow-up was 14 mo. The median follow-up among the 227 nivolumab-randomized and 196 everolimus-randomized patients who had not died at data cutoff was 22 mo (interquartile range [IQR] 20-25) and $22 \mathrm{mo}$ (IQR 20-25). At data cutoff (June 2015), 67 of 406 nivolumabtreated and 28 of 397 everolimus-treated patients continued to receive treatment. The number who continued to receive treatment in each subgroup is shown in Supplementary Fig. 1. Consistent with the overall population, the primary reason for discontinuation in all subgroups was disease progression.

The baseline disease characteristics of patients by subgroups were generally similar between nivolumab and everolimus (Supplementary Table 1).

\subsection{OS by prognostic risk group}

In an assessment of OS by favorable, intermediate, and poor MSKCC risk groups, median OS was longer in both arms in patients with better MSKCC scores (Fig. 1A, Fig. 2A-C). Among patients with poor risk who received nivolumab, median OS was almost double compared with everolimus (hazard ratio 0.48; Fig. 1A, Fig. 2C). Results for OS by IMDC risk group were consistent with those for MSKCC risk group (Supplementary Fig. 2A-C; Fig. 1A). The mortality rate at
Table 1 - Distribution of randomized patients within each subgroup

\begin{tabular}{|c|c|c|}
\hline \multirow[t]{2}{*}{ Subgroup ${ }^{a}$} & \multicolumn{2}{|c|}{ Patients, $n(\%)$} \\
\hline & $\begin{array}{l}\text { Nivolumab } \\
(N=410)\end{array}$ & $\begin{array}{c}\text { Everolimus } \\
(N=411)\end{array}$ \\
\hline \multicolumn{3}{|l|}{ MSKCC risk score } \\
\hline Favorable & $137(33)$ & $145(35)$ \\
\hline Intermediate & $193(47)$ & $192(47)$ \\
\hline Poor & $79(19)$ & $74(18)$ \\
\hline \multicolumn{3}{|l|}{ IMDC risk score } \\
\hline Favorable & $55(13)$ & $70(17)$ \\
\hline Intermediate & $242(59)$ & $241(59)$ \\
\hline Poor & $96(23)$ & $83(20)$ \\
\hline Not reported & $17(4)$ & $17(4)$ \\
\hline \multicolumn{3}{|l|}{ Age group } \\
\hline$<65 \mathrm{yr}$ & 257 (63) & $240(58)$ \\
\hline$\geq 65 \mathrm{yr}$ & $153(37)$ & $171(42)$ \\
\hline \multicolumn{3}{|c|}{ Number of sites of metastases } \\
\hline 1 & $68(17)$ & $71(17)$ \\
\hline$\geq 2$ & $341(83)$ & $338(82)$ \\
\hline \multicolumn{3}{|l|}{ Site of metastases } \\
\hline Bone & $76(19)$ & $70(17)$ \\
\hline Liver & $100(24)$ & $87(21)$ \\
\hline Lung & $278(68)$ & $273(66)$ \\
\hline \multicolumn{3}{|l|}{ Prior therapy ${ }^{b}$} \\
\hline Sunitinib & $257(63)$ & $261(64)$ \\
\hline Pazopanib & $126(31)$ & $136(33)$ \\
\hline Interleukin-2 & $42(10)$ & $37(9)$ \\
\hline \multicolumn{3}{|c|}{ Time on first-line therapy } \\
\hline$<6 \mathrm{mo}$ & $110(27)$ & $130(32)$ \\
\hline$\geq 6 \mathrm{mo}$ & $300(73)$ & $281(68)$ \\
\hline \multicolumn{3}{|c|}{ Prior antiangiogenic therapies } \\
\hline 1 & $317(77)$ & $312(76)$ \\
\hline 2 & $90(22)$ & $99(24)$ \\
\hline \multicolumn{3}{|c|}{$\begin{array}{l}\text { IMDC = International Metastatic Renal Cell Carcinoma Database } \\
\text { Consortium; MSKCC }=\text { Memorial Sloan Kettering Cancer Center. } \\
\text { a Analysis based on case report form data. } \\
\text { b Patients may have received more than one prior therapy. }\end{array}$} \\
\hline
\end{tabular}

12 mo for all subgroups was lower with nivolumab compared with everolimus and was particularly striking in the poor MSKCC risk group (Fig. 1A).

\subsection{OS by age group}

Median OS in patients aged $<65 \mathrm{yr}$ was 26.7 mo (95\% CI 21.8-NR) with nivolumab and 19.9 mo (95\% CI 17.4-NR) with everolimus. Median OS in patients aged $\geq 65 \mathrm{yr}$ was $23.6 \mathrm{mo}$ (95\% CI 18.2-NR) with nivolumab and $18.5 \mathrm{mo}$ (95\% CI 16.4-21.6) with everolimus.

\subsection{OS by number and site of metastases}

Median OS in patients with one site of metastasis was NR with nivolumab and $29.0 \mathrm{mo}$ (95\% CI NR) with everolimus (Supplementary Fig. 3A, Fig. 1A). In patients with at least two sites of metastases, median OS was 22.2 mo (95\% CI 19.1-26.7) with nivolumab and $17.6 \mathrm{mo}$ (95\% CI 15.6-19.8) with everolimus (Supplementary Fig. 3B, Fig. 1A).

Median OS in patients with bone metastases was $18.5 \mathrm{mo}$ (95\% CI 10.2-NR) with nivolumab and $13.8 \mathrm{mo}$ (95\% CI 7.0-16.4) with everolimus (Supplementary Fig. 4A, Fig. 1A). Median OS in patients with liver metastases was 


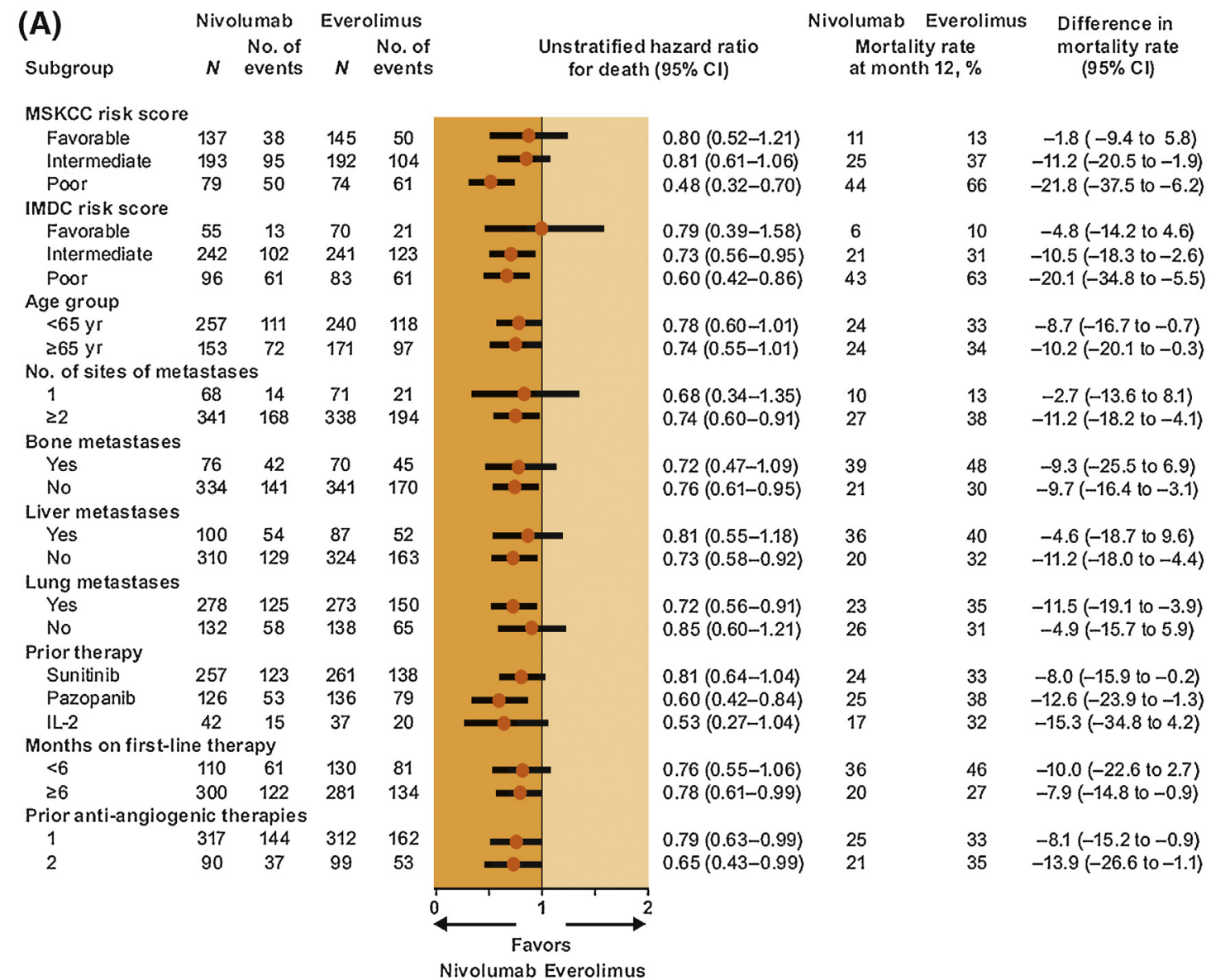

(B)

\begin{tabular}{|c|c|c|c|c|c|}
\hline \multirow[b]{2}{*}{ Subgroup } & \multicolumn{2}{|c|}{ Nivolumab } & \multicolumn{2}{|c|}{ Everolimus } & \multirow{2}{*}{$\begin{array}{l}\text { ORR difference } \\
\qquad(95 \% \mathrm{Cl})\end{array}$} \\
\hline & ORR, \% & $95 \% \mathrm{Cl}$ & ORR, \% & $95 \% \mathrm{Cl}$ & \\
\hline \multicolumn{6}{|c|}{ MSKCC risk group } \\
\hline Favorable & 24 & $17-32$ & 8 & $4-13$ & - \\
\hline Intermediate & 25 & $19-32$ & 5 & $2-9$ & - \\
\hline Poor & 27 & $17-38$ & 3 & $0.3-9$ & \\
\hline \multicolumn{6}{|l|}{ Age group } \\
\hline$<65 \mathrm{yr}$ & 23 & $18-29$ & 5 & $3-9$ & \\
\hline$\geq 65 \mathrm{yr}$ & 29 & $22-37$ & 6 & $3-11$ & \\
\hline \multicolumn{6}{|c|}{ No. of sites of metastases } \\
\hline 1 & 32 & $22-45$ & 9 & $3-18$ & \\
\hline$\geq 2$ & 24 & $19-29$ & 5 & $3-8$ & $-\infty$ \\
\hline \multicolumn{6}{|c|}{ Bone metastases } \\
\hline Yes & 26 & $17-38$ & 6 & $2-14$ & 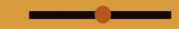 \\
\hline No & 25 & $20-30$ & 5 & $3-8$ & $-\infty$ \\
\hline \multicolumn{6}{|c|}{ Liver metastases } \\
\hline Yes & 21 & $14-30$ & 3 & $1-10$ & 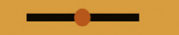 \\
\hline No & 27 & $22-32$ & 6 & $4-9$ & - \\
\hline \multicolumn{6}{|c|}{ Lung metastases } \\
\hline Yes & 27 & $22-33$ & 5 & $3-8$ & $=$ \\
\hline No & 21 & $15-29$ & 7 & 3-12 & \\
\hline \multicolumn{6}{|l|}{ Prior therapy } \\
\hline Sunitinib & 23 & $18-28$ & 6 & $4-10$ & - \\
\hline Pazopanib & 28 & $20-37$ & 3 & $1-7$ & \\
\hline IL-2 & 14 & $5-29$ & 3 & $0.1-14$ & \\
\hline \multicolumn{6}{|c|}{ Months on first-line therapy } \\
\hline$<6$ & 26 & $18-35$ & 5 & 2-11 & \\
\hline$\geq 6$ & 25 & $20-30$ & 5 & $3-9$ & - \\
\hline \multicolumn{6}{|c|}{ Prior anti-angiogenic therapies } \\
\hline \multirow{3}{*}{$\begin{array}{l}1 \\
2\end{array}$} & 24 & $20-29$ & 5 & $3-9$ & - \\
\hline & 28 & $19-38$ & 5 & $2-11$ & \\
\hline & & & & & $\begin{array}{lll}10 & 20 & 30\end{array}$ \\
\hline
\end{tabular}

Fig. 1 - Forest plots of (A) overall survival and (B) objective response rate by baseline characteristics (risk group, age group, number and sites of metastases, prior therapy). CI = confidence interval; IL-2 = interleukin-2; IMDC = International Metastatic Renal Cell Carcinoma Database Consortium; MSKCC = Memorial Sloan Kettering Cancer Center; $O R R=$ overall response rate; OS = overall survival. 
(A)

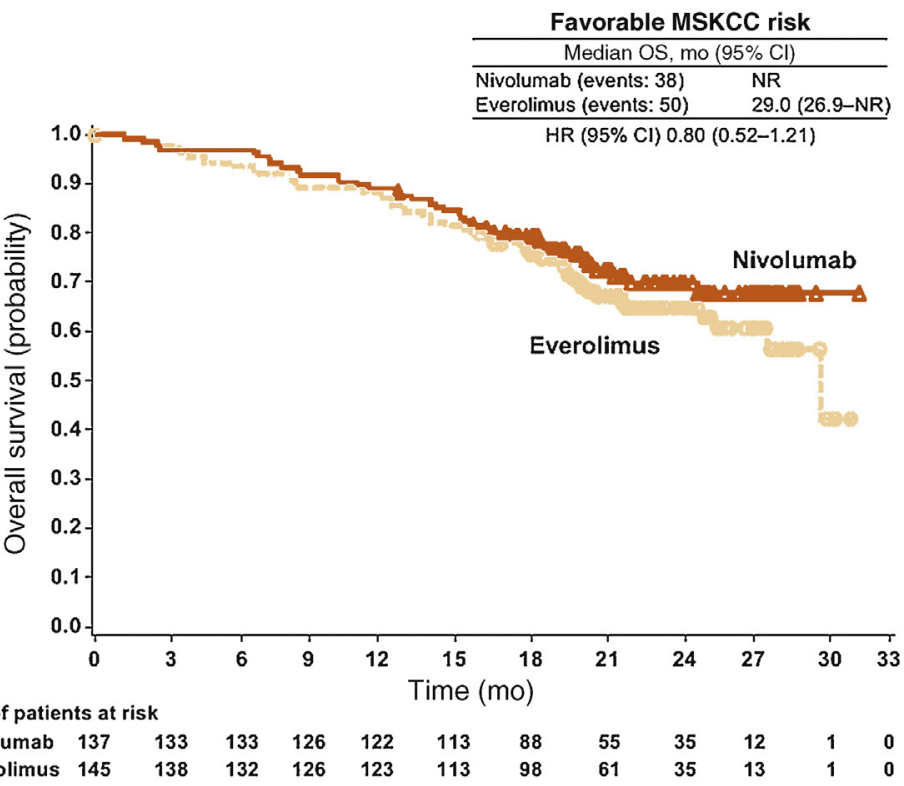

(B)

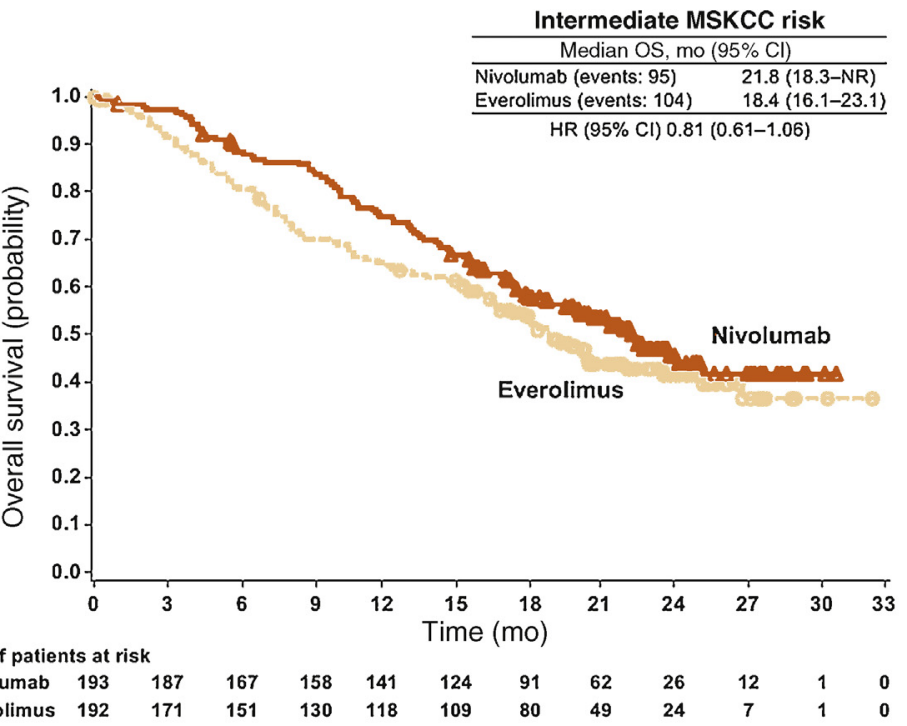

(C)

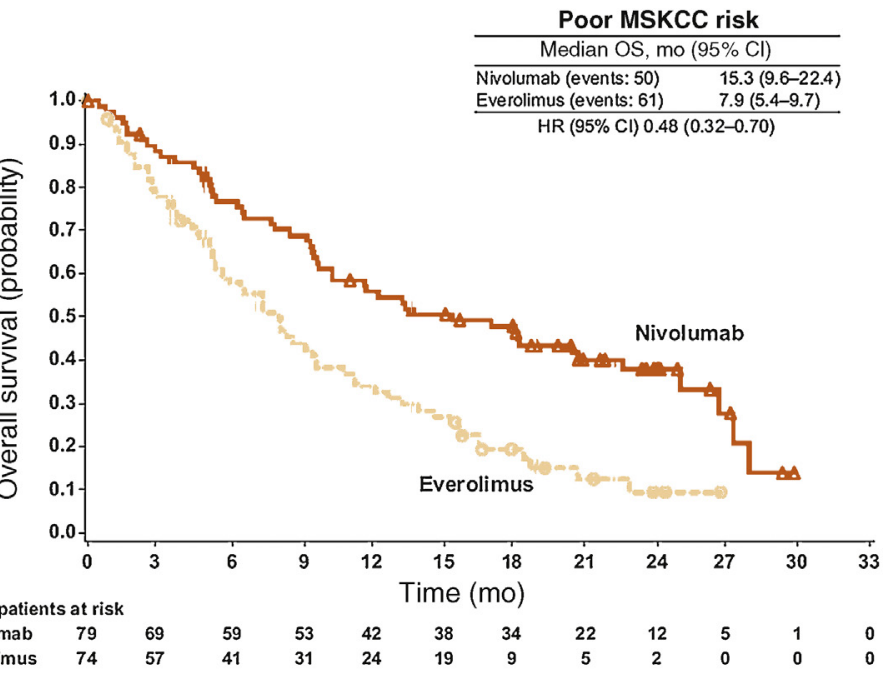

Fig. 2 - Kaplan-Meier curves for overall survival by (A) favorable, $(B)$ intermediate, and (C) poor MSKCC risk group. $\mathrm{CI}=$ confidence interval; HR = hazard ratio; MSKCC = Memorial Sloan Kettering Cancer Center; $N R=$ not reached; $0 S=$ overall survival. 
(A)

Prior sunitinib $\frac{\text { Median OS, mo }(95 \% \mathrm{Cl})}{\text { Nivolumab (events: } 123)}$

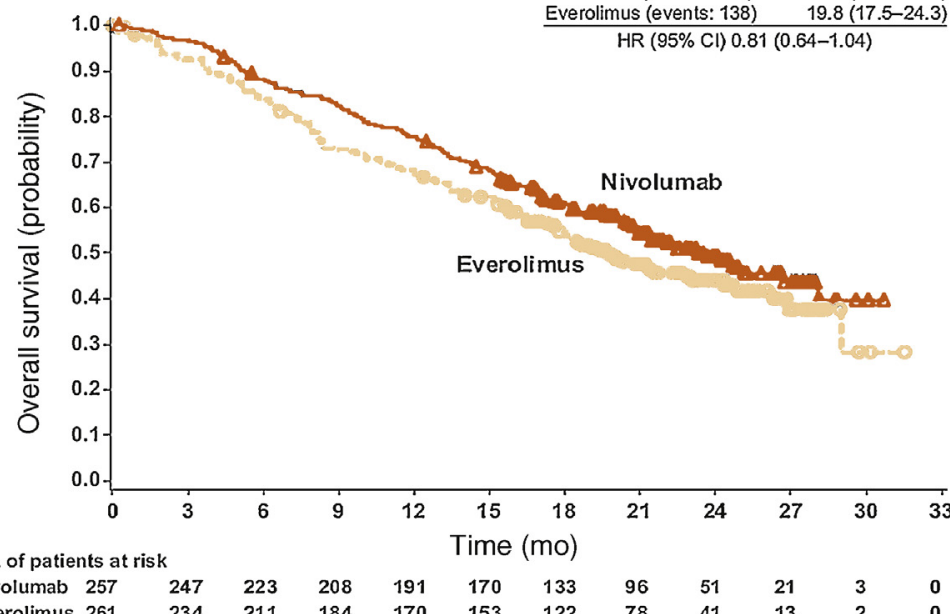

$\begin{array}{lllllllllllll}\text { Nivolumab } & 257 & 247 & 223 & 208 & 191 & 170 & 133 & 96 & 51 & 21 & 3 & 0 \\ \text { Everolimus } & 261 & 234 & 211 & 184 & 170 & 153 & 122 & 78 & 41 & 13 & 2 & 0\end{array}$

(B)

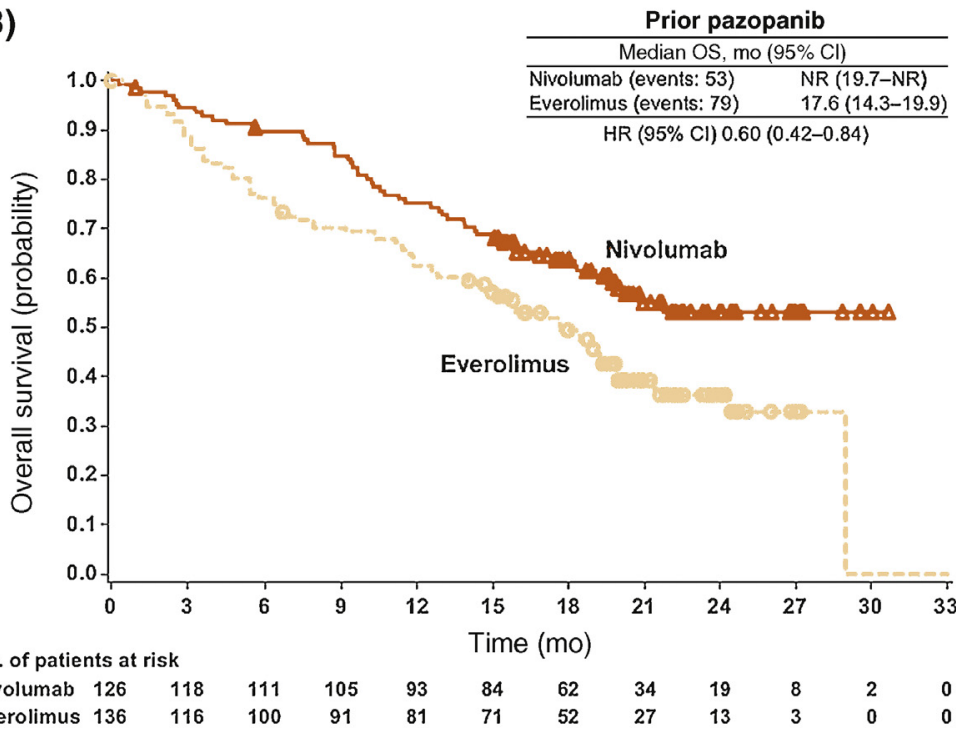

(C)

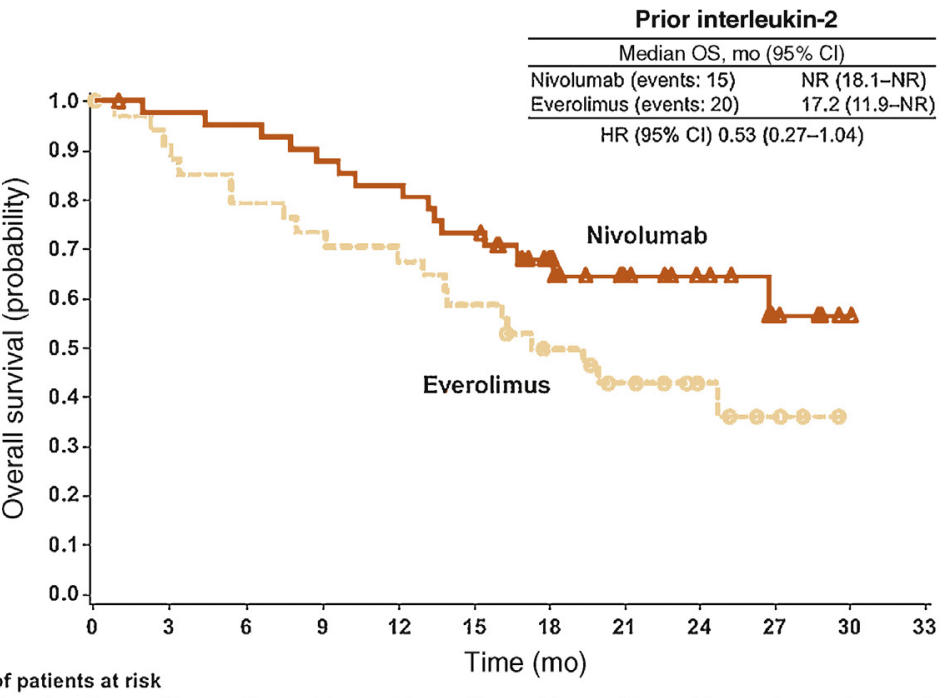

No. of patients at risk

$\begin{array}{lllllllllllll}\text { Nivolumab } & 42 & 40 & 39 & 36 & 34 & 30 & 21 & 14 & 10 & 5 & 1 & 0\end{array}$

Fig. 3 - Kaplan-Meier curves for overall survival by (A) prior sunitinib therapy, (B) prior pazopanib therapy, and (C) prior interleukin-2 therapy. $\mathrm{CI}=$ confidence interval; $\mathrm{HR}=$ hazard ratio; $\mathrm{NR}=$ not reached; $\mathrm{OS}=$ overall survival. 
18.3 mo (95\% CI 13.4-26.7) with nivolumab and 16.0 mo (95\% CI 8.4-21.6) with everolimus (Supplementary Fig. 4B; Fig. 1A). Median OS in patients with lung metastases was $25.0 \mathrm{mo}$ (95\% CI 20.4-NR) with nivolumab and $18.7 \mathrm{mo}$ (95\% CI 16.2-21.2) with everolimus (Supplementary Fig. S4C, Fig. 1A).

\subsection{OS by prior therapy}

Median OS in patients with prior sunitinib or pazopanib or interleukin-2 therapy was longer with nivolumab than with everolimus or was not yet reached for nivolumab (Fig. 3, Fig. 1A; reported previously for sunitinib and pazopanib in the nivolumab arm [14]).
When the overall treated population was divided according to first-line therapy duration, median OS was longer for both treatment arms in patients with $\geq 6$ mo compared with $<6$ mo on first-line therapy and was longer with nivolumab compared with everolimus (Fig. 4, Fig. 1A).

Median OS in patients with one prior antiangiogenic therapy was $23.6 \mathrm{mo}$ (95\% CI 20.8-NR) with nivolumab and 19.9 mo (95\% CI 17.7-24.7) with everolimus (Fig. 1A).

\subsection{Interaction test for each subgroup}

An interaction test of treatment and each subgroup with OS revealed a significant interaction for MSKCC risk group

\section{(A)}

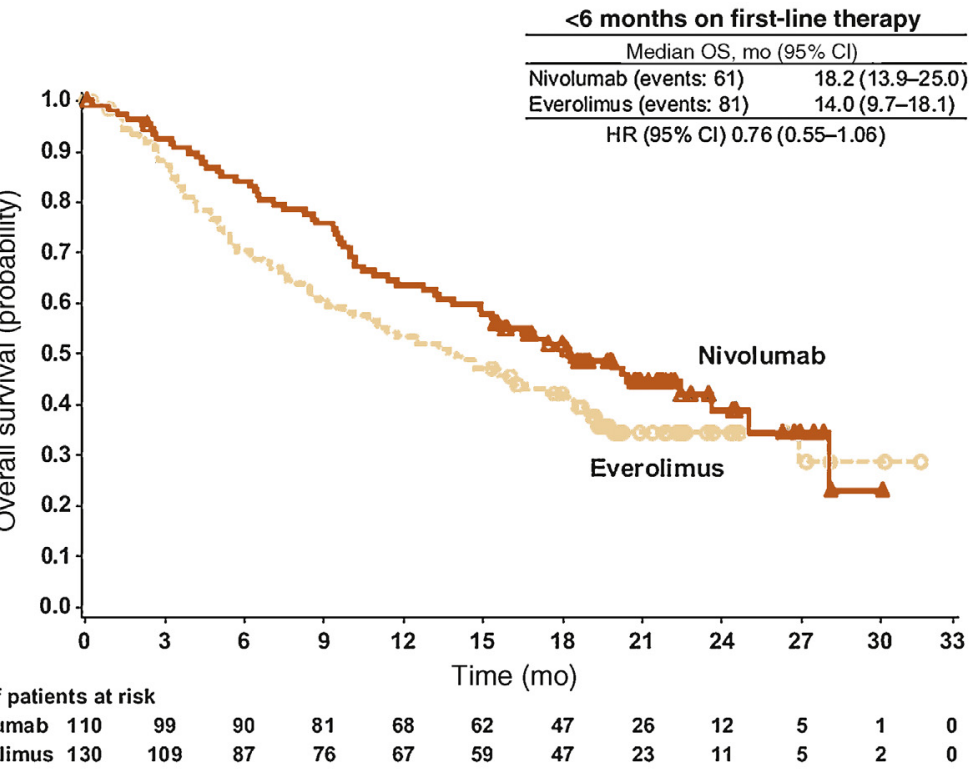

(B)

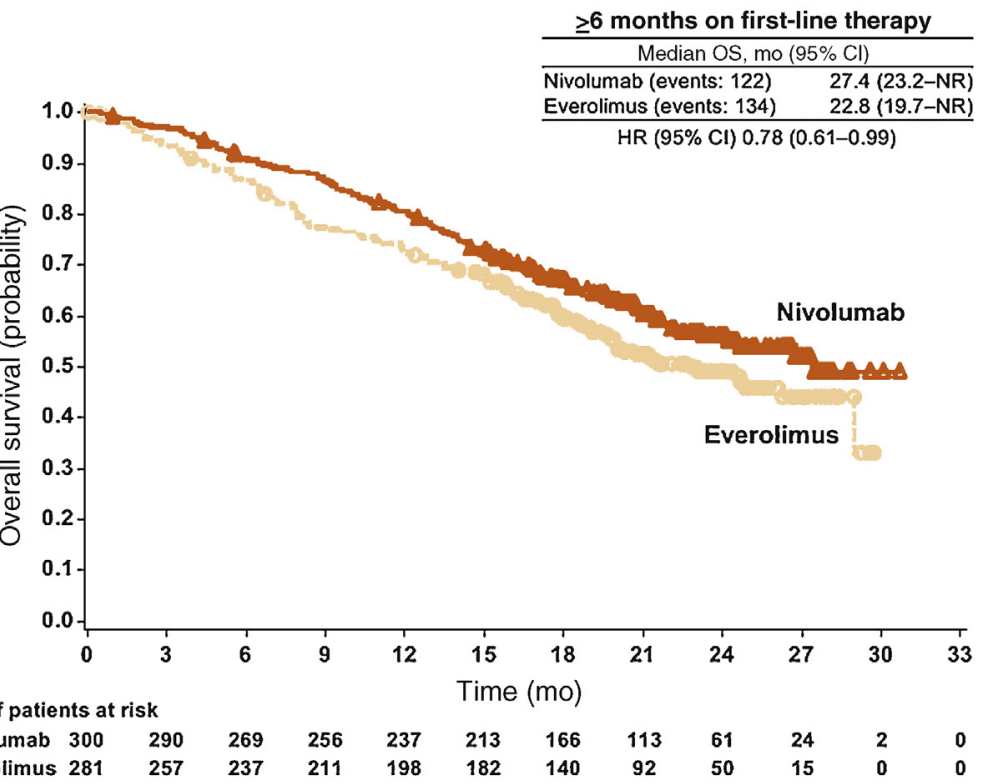


Table 2 - Summary of treatment-related adverse events for patients within each subgroup

\begin{tabular}{|c|c|c|c|c|}
\hline \multirow[t]{3}{*}{ Subgroup } & \multicolumn{4}{|c|}{ Treatment-related adverse events, $n$ (\%) } \\
\hline & \multicolumn{2}{|c|}{ Nivolumab } & \multicolumn{2}{|c|}{ Everolimus } \\
\hline & Any grade & Grade 3-4 & Any grade & Grade 3-4 \\
\hline All treated patients & $319(79)$ & $76(19)$ & $349(88)$ & $145(37)$ \\
\hline \multicolumn{5}{|l|}{ MSKCC risk score } \\
\hline Favorable & $116(85)$ & $21(15)$ & $131(93)$ & $56(40)$ \\
\hline Intermediate & $143(74)$ & $36(19)$ & $160(87)$ & $63(34)$ \\
\hline Poor & $60(78)$ & $19(25)$ & $58(80)$ & $26(36)^{a}$ \\
\hline \multicolumn{5}{|l|}{ IMDC risk score } \\
\hline Favorable & $46(84)$ & $11(20)$ & $67(99)$ & $28(41)$ \\
\hline Intermediate & $189(78)$ & $41(17)$ & $202(87)$ & $83(36)^{b}$ \\
\hline Poor & $71(76)$ & $21(22)$ & $64(80)$ & $26(33)^{b}$ \\
\hline Not reported & $13(81)$ & $3(19)$ & $16(100)$ & $8(50)$ \\
\hline \multicolumn{5}{|l|}{ Age group } \\
\hline$<65 \mathrm{yr}$ & $200(79)$ & $51(20)$ & $199(86)$ & $81(35)^{a}$ \\
\hline$\geq 65 \mathrm{yr}$ & $119(78)$ & $25(16)$ & $150(90)$ & $64(39)$ \\
\hline \multicolumn{5}{|c|}{ Number of sites of metastases } \\
\hline 1 & $57(85)$ & $10(15)$ & $62(90)$ & $23(33)$ \\
\hline$\geq 2$ & $262(77)$ & $66(19)$ & $287(88)$ & $122(37)^{\mathrm{a}}$ \\
\hline \multicolumn{5}{|l|}{ Site of metastases } \\
\hline Bone & $51(67)$ & $13(17)$ & $50(75)$ & $13(19)$ \\
\hline Liver & $78(80)$ & $22(22)$ & $72(85)$ & $31(36)^{b}$ \\
\hline Lung & $215(78)$ & $46(17)$ & $232(88)$ & $97(37)^{\mathrm{b}}$ \\
\hline \multicolumn{5}{|l|}{ Prior therapy } \\
\hline Sunitinib & $197(78)$ & $46(18)$ & $220(88)$ & $89(35)^{a}$ \\
\hline Pazopanib & $103(82)$ & $24(19)$ & $114(89)$ & $45(35)^{\mathrm{b}}$ \\
\hline Interleukin-2 & $37(88)$ & $8(19)$ & $30(88)$ & $13(38)$ \\
\hline \multicolumn{5}{|c|}{ Time on first-line therapy } \\
\hline$<6 \mathrm{mo}$ & $85(79)$ & $24(22)$ & $109(88)$ & $44(35)^{b}$ \\
\hline$\geq 6 \mathrm{mo}$ & $234(78)$ & $52(17)$ & $240(88)$ & $101(37)^{b}$ \\
\hline \multicolumn{5}{|c|}{ Prior antiangiogenic therapies } \\
\hline 1 & $240(76)$ & $61(19)$ & $264(87)$ & $111(37)^{b}$ \\
\hline 2 & $76(85)$ & $15(17)$ & $85(90)$ & $34(36)^{\mathrm{b}}$ \\
\hline
\end{tabular}

$(p=0.0475)$. There was no evidence of an interaction for other subgroups.

\subsection{ORR for each subgroup}

ORR favored nivolumab over everolimus in all subgroups, most notably in patients with poor MSKCC risk, aged at least $65 \mathrm{yr}$, one site of metastasis, lung metastases, prior pazopanib therapy, and two prior antiangiogenic therapies (Fig. 1B). There was no evidence of an interaction between treatment and each subgroup with ORR.

\subsection{Safety for each subgroup}

The incidence of grade 3 or 4 TRAEs was lower in the nivolumab arm compared with the everolimus arm across subgroups (Table 2). The incidence of grade 3 or 4 TRAEs in the nivolumab arm was half or less than half the incidence observed with everolimus in the following subgroups: favorable MSKCC risk (15\% vs 40\%); favorable IMDC risk ( $20 \%$ vs $41 \%$ ); intermediate IMDC risk (17\% vs 36\%); at least $65 \mathrm{yr}$ of age (16\% vs 39\%); one site of metastasis (15\% vs $33 \%)$; lung metastases (17\% vs $37 \%$ ); prior interleukin-2 therapy (19\% vs 38\%); at least 6 mo on first-line therapy
(17\% vs $37 \%$ ); and two prior antiangiogenic therapies $(17 \%$ vs $36 \%$; Table 2). There was no evidence of an interaction between treatment and each subgroup with any TRAE.

\section{Discussion}

With a minimum follow-up of 14 mo in previously treated patients with aRCC, OS and ORR favored nivolumab over everolimus for multiple subgroups. Within the nivolumab arm, ORR for most subgroups was similar and consistent with the overall ORR reported previously [7].

Median OS was greater and mortality rate was lower with nivolumab than with everolimus in all MSKCC risk groups, with the largest difference in patients with poor risk. The small number of events and short duration of follow-up in the favorable risk group limited the ability to observe robust OS differences between arms. The large difference for poor-risk patients suggests that further investigation of the characteristics of these patients, such as tumor biology, is needed to better understand this finding. One explanation, yet to be formally analyzed, is the potential presence of a higher mutational load in poor-risk patients, a phenomenon that in some cases has been correlated with better efficacy of PD-1 inhibitors. This is 
possibly because of the higher levels of lymphocytes expressing immune inhibitory signals in the tumor microenvironment that can be targeted by PD- 1 inhibitors $[15,16]$. The improved OS with nivolumab versus everolimus in patients with liver or bone metastases, which are associated with high incidence and poor prognosis $[3,17,18]$, further demonstrates that patients with poor prognostic features benefit from treatment with nivolumab.

There are some suggestions that response to nivolumab may be influenced by prior effect of tyrosine kinase inhibitors on the tumor microenvironment or their potential immune effect $[14,19,20]$. Although this study did not directly address the tumor microenvironment or the sequence of therapy, we did observe that OS was improved with nivolumab for all three prior therapies evaluated (sunitinib, pazopanib, and interleukin-2). Interleukin-2 is currently indicated in first-line treatment for a select group of patients with excellent performance status and normal organ function [21]. The small number of patients with prior interleukin-2 treatment enrolled and clinical selection criteria that impact decisions to treat with interleukin-2 preclude substantial clinical inferences. However, the favorable hazard ratio for nivolumab versus everolimus may imply a special impact of immune system manipulation by immunotherapies such as interleukin-2 that may result in benefit from subsequent treatment with an immune checkpoint inhibitor.

the superior OS for nivolumab was maintained regardless of the duration of first-line therapy, suggesting that a switch from a tyrosine kinase inhibitor to nivolumab, either for a lack of response or toxicity (subgroup with $<6$ mo of first-line therapy) or following potentially successful firstline therapy (subgroup with $\geq 6$ mo of first-line therapy) provides a survival benefit.

The incidence of all-grade and grade 3 or 4 TRAEs in each subgroup was lower with nivolumab than with everolimus and was similar to TRAEs noted in the overall population [7]. Notably, both younger and older patients had a similar incidence of grade 3 or 4 TRAEs. This observation, coupled with the improved OS with nivolumab versus everolimus in younger and older patients, suggests that older age did not preclude clinical benefit.

Some limitations should be noted, including the post hoc nature of the analyses and the differing sample sizes within subgroups. The small sample size in some subgroups limits the interpretation of those results and may have contributed to the large range for some $95 \%$ CIs associated with hazard ratios for death and difference in ORR. Analyses with more patients are needed to validate those results. Furthermore, in the subgroup analyses of individual sites of metastases, some patients with multiple sites of metastases were represented in more than one subgroup, which complicates interpretation of OS by number of sites of metastases.

The broad benefit of nivolumab versus everolimus in terms of OS and ORR with respect to patient demographics, clinical characteristics, and prior therapies may provide additional insight that will allow clinicians to make informed decisions. With the increasing number of therapies available to treat aRCC, predicting outcomes, possibly through the development of nomograms based on baseline disease characteristics and prior therapies, will help to increase the individualized approach to treatment in an effort to improve survival [22]. Further research should focus on the development of predictive models of outcomes more aligned with immunotherapy in accordance with findings from this study.

In conclusion, consistent with the benefit demonstrated in the overall population from CheckMate 025 [7], a trend for OS and ORR benefit with nivolumab versus everolimus was observed for multiple subgroups, including prognostic risk categories, age, number and sites of metastases, and prior therapies, without specific safety concerns. Efficacy with nivolumab is notable in patients with poor risk features, including those in the poor MSKCC risk group, those with bone metastases, and those with more than one site of metastasis. These results further support the use of nivolumab as a new standard of care $[21,23,24]$ for a broad range of patients with previously treated aRCC.

Presented, in part, at the Annual Society of Clinical Oncology-Genitourinary Cancers Symposium, San Francisco, California, USA, January 7-9, 2016.

Author contributions: Bernard Escudier had full access to all the data in the study and takes responsibility for the integrity of the data and the accuracy of the data analysis.

Study concept and design: Motzer, Waxman.

Acquisition of data: Waxman, Zhao.

Analysis and interpretation of data: All authors.

Drafting of the manuscript: Escudier, Motzer.

Critical revision of the manuscript for important intellectual content: All authors.

Statistical analysis: Zhao.

Obtaining funding: None.

Administrative, technical, or material support: None.

Supervision: None.

Other: None.

Financial disclosures: Bernard Escudier certifies that all conflicts of interest, including specific financial interests and relationships and affiliations relevant to the subject matter or materials discussed in the manuscript (eg, employment/affiliation, grants or funding, consultancies, honoraria, stock ownership or options, expert testimony, royalties, or patents filed, received, or pending), are the following: Frede Donskov has received institutional research funding from Novartis, GlaxoSmithKline, and Pfizer. Bernard Escudier has received honoraria from Bayer, Novartis, Pfizer, Bristol-Myers Squibb, and Exelixis. Thomas Gauler owns stock in Bayer AG; has received consulting or advisory fees from MSD, Merck Serono, Novartis, and Bristol-Myers Squibb; and has received travel and accommodation expenses from Boehringer Ingelheim, Merck Serono, Novartis, MSD, Bayer Healthcare, Hoffmann-La Roche, and Bristol-Myers Squibb. Saby George has an immediate family member employed by Amgen, and has received research funding from BristolMyers Squibb, Novartis, Pfizer, Bayer, Acceleron, and Agensys. Howard Gurney has received consulting or advisory fees from Astellas, BristolMyers Squibb, Novartis, and Pfizer. Hans Hammers has received consulting or advisory fees from Bristol-Myers Squibb, Exelixis, Pfizer, and Cerulean, and research funding from SFJ, Bristol-Myers Squibb, Exelixis, Newlink, Pfizer, GlaxoSmithKline, and Tracon. David McDermott has received consulting or advisory fees from Bristol-Myers Squibb, Merck, Genentech, Novartis, Pfizer, Eisai, and Exelixis, and institutional research funding from Promethius Labs. Robert Motzer has received 
consulting or advisory fees from Novartis, Eisai, and Pfizer, and research funding from Exelixis, Bristol-Myers Squibb, Novartis, GlaxoSmithKline, Eisai, and Pfizer. Katrina Peltola has received advisory fees from MSD, Bristol-Myers Squibb, Lilly, Sanofi, Astellas, Pfizer, and Novartis, and travel compensation from Pierre Fabre and Bristol-Myers Squibb. Elizabeth Plimack has received consulting or advisory fees from Merck, Dendreon, Novartis, Bristol-Myers Squibb, Pfizer, GlaxoSmithKline, Acceleron Pharma, and Genentech/Roche, and institutional research funding from Merck, Bristol-Myers Squibb, GlaxoSmithKline, Acceleron Pharma, Dendreon, Lilly, and AstraZeneca, and has an institutional interest in US Patent No. 14/588.503. Giuseppe Procopio has received grants from Bayer and personal fees from Astellas, Bristol-Myers Squibb, Janssen, Novartis, and Pfizer. Padmanee Sharma has received leadership fees from Kite Pharma, Jounce, and Evelo; consultant or advisor fees from BristolMyers Squibb, GlaxoSmithKline, AstraZeneca, and Amgen; investigator fees from Bristol-Myers Squibb, GlaxoSmithKline, and AstraZeneca. She has investment interests in Jounce, Kite Pharma, Evelo, and owns a patent licensed to Jounce. Her spouse has received leadership fees from Kite Pharma, Jounce, and Neon; has investment interests in Jounce, Kite, and Neon; and has an interest in patents licensed to Bristol-Myers Squibb, Jounce, and Merck. Jeffrey Sosman has received honoraria and consulting or advisory fees from Novartis, Genentech, Array, and Merck; and research funding from Bristol-Myers Squibb, Biomedical Valley Development, and GlaxoSmithKline. Scott Tykodi has received consulting or advisory fees from Prometheus Laboratories and Amgen, and institutional research funding from Genentech, Bristol-Myers Squibb, Exelixis, Argos Therapeutics, GlaxoSmithKline, and Prometheus. An immediate family member of John Wagstaff has received honoraria from Roche, Bristol-Myers Squibb, Pfizer, Novartis, and MSD; consulting or advisory fees from Roche, BristolMyers Squibb, Pfizer, Novartis, and MSD; and travel and accommodation expenses from Bristol-Myers Squibb and MSD. Ian Waxman and Huanyu Zhao are employed by and hold stock in Bristol-Myers Squibb. Daniel Castellano, Sandhya Srinivas, and Takeshi Ueda have nothing to disclose.

Funding/Support and role of the sponsor: This study was sponsored by Bristol-Myers Squibb and Ono Pharmaceutical Company Limited. The sponsors contributed to the design and conduct of the study; collection, management, analysis, and interpretation of the data; and preparation, review, and approval of the manuscript in collaboration with the investigators and authors of this report.

Acknowledgments: We thank the patients and their families, as well as the investigators and participating study teams, for making this study possible. Medical writing assistance was provided by Jennifer Granit of PPSI (a PAREXEL company) and was funded by Bristol-Myers Squibb.

\section{Appendix A. Supplementary data}

Supplementary data associated with this article can be found, in the online version, at http://dx.doi.org/10.1016/j. eururo.2017.02.010.

\section{References}

[1] Heng DY, Xie W, Regan MM, et al. Prognostic factors for overall survival in patients with metastatic renal cell carcinoma treated with vascular endothelial growth factor-targeted agents: results from a large, multicenter study. J Clin Oncol 2009;27:5794-9.

[2] Motzer RJ, Bacik J, Schwartz LH, et al. Prognostic factors for survival in previously treated patients with metastatic renal cell carcinoma. J Clin Oncol 2004;22:454-63.

[3] McKay RR, Kroeger N, Xie W, et al. Impact of bone and liver metastases on patients with renal cell carcinoma treated with targeted therapy. Eur Urol 2014;65:577-84.
[4] Calvo E, Escudier B, Motzer RJ, et al. Everolimus in metastatic renal cell carcinoma: Subgroup analysis of patients with 1 or 2 previous vascular endothelial growth factor receptor-tyrosine kinase inhibitor therapies enrolled in the phase III RECORD-1 study. Eur J Cancer 2012;48:333-9.

[5] Escudier B, Michaelson MD, Motzer RJ, et al. Axitinib versus sorafenib in advanced renal cell carcinoma: subanalyses by prior therapy from a randomised phase III trial. $\mathrm{Br} \mathrm{J}$ Cancer 2014;110:2821-8.

[6] Taccoen X, Valeri A, Descotes JL, et al. Renal cell carcinoma in adults 40 years old or less: young age is an independent prognostic factor for cancer-specific survival. Eur Urol 2007;51:980-7.

[7] Motzer RJ, Escudier B, McDermott DF, et al. Nivolumab versus everolimus in advanced renal-cell carcinoma. N Engl J Med 2015;373:1803-13.

[8] Opdivo (nivolumab) injection [prescribing information]. Princeton, NJ: Bristol-Myers Squibb; 2015.

[9] Eisenhauer EA, Therasse P, Bogaerts J, et al. New response evaluation criteria in solid tumours: revised RECIST guideline (version 1.1). Eur J Cancer 2009;45:228-47.

[10] Kaplan EL, Meier P. Nonparametric estimation from incomplete observations. J Am Stat Assoc 1958;53:457-81.

[11] Brookmeyer R, Crowley J. A confidence interval for the median survival time. Biometrics 1982;38:29-41.

[12] Cox DR. Regression models and life tables. J R Stat Soc Series B Stat Methodol 1972;34:187-220.

[13] Fagerland MW, Lydersen S, Laake P. Recommended confidence intervals for two independent binomial proportions. Stat Methods Med Res 2015;24:224-54.

[14] Motzer RJ, Escudier B, Choueiri TK. Treatment of advanced renalcell carcinoma. N Engl J Med 2016;374:888-90.

[15] Rizvi NA, Hellmann MD, Snyder A, et al. Cancer immunology. Mutational landscape determines sensitivity to PD-1 blockade in non-small cell lung cancer. Science 2015;348:124-8.

[16] Le DT, Uram JN, Wang H, et al. PD-1 blockade in tumors with mismatch-repair deficiency. N Engl J Med 2015;372:2509-20.

[17] Grimes NG, Devlin JM, Dunne DF, et al. A systematic review of the role of hepatectomy in the management of metastatic renal cell carcinoma. Eur J Surg Oncol 2014;40:1622-8.

[18] Vrdoljak E, Rini B, Schmidinger M, et al. Bisphosphonates and vascular endothelial growth factor-targeted drugs in the treatment of patients with renal cell carcinoma metastatic to bone. Anticancer Drugs 2013;24:431-40.

[19] Stewart GD, O'Mahony FC, Laird A, et al. Sunitinib treatment exacerbates intratumoral heterogeneity in metastatic renal cancer. Clin Cancer Res 2015;21:4212-23.

[20] Guislain A, Gadiot J, Kaiser A, et al. Sunitinib pretreatment improves tumor-infiltrating lymphocyte expansion by reduction in intratumoral content of myeloid-derived suppressor cells in human renal cell carcinoma. Cancer Immunol Immunother 2015;64:1241-50.

[21] National Comprehensive Cancer Network. NCCN clinical practice guidelines in oncology: kidney cancer. Version 2.2016. www.nccn. org/professionals/physician_gls/f_guidelines.asp.

[22] Kattan MW, Reuter V, Motzer RJ, Katz J, Russo P. A postoperative prognostic nomogram for renal cell carcinoma. J Urol 2001;166: 63-7.

[23] Powles T, Staehler M, Ljungberg B, et al. Updated EAU guidelines for clear cell renal cancer patients who fail VEGF targeted therapy. Eur Urol 2016;69:4-6.

[24] Escudier B, Porta C, Schmidinger M, et al. Renal cell carcinoma: ESMO Clinical Practice Guidelines for diagnosis, treatment and follow-up. Ann Oncol 2016;27:v58-68. 